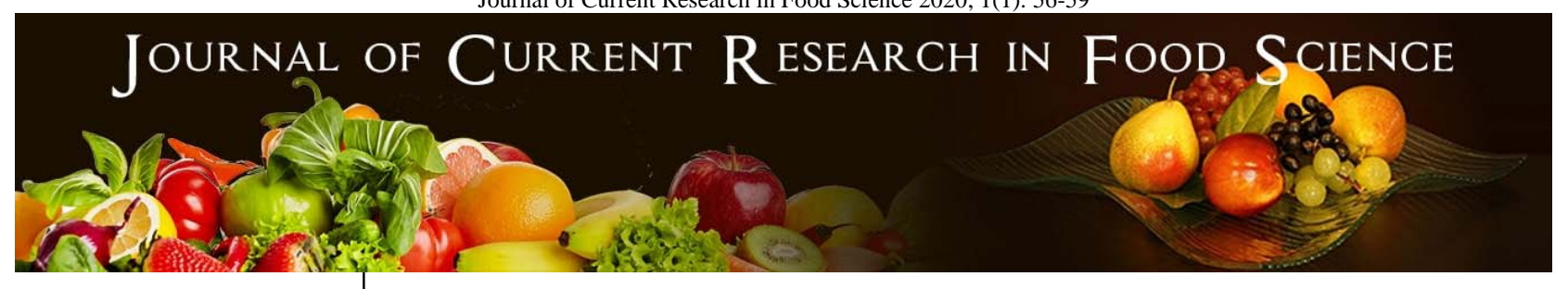

E-ISSN: 2709-9385 P-ISSN: 2709-9377 JCRFS 2020; 1(1): 56-59 (C) 2020 JCRFS

www.foodresearchjournal.com Received: 01-04-2020 Accepted: 05-05-2020

Swati Suman

Research Scholar, University Department of Home Science, Ranchi University, Ranchi, Jharkhand, India

Seema Dey

Associate Professor, University Department of Home Science, Ranchi University, Ranchi, Jharkhand, India
Corresponding Author: Swati Suman Research Scholar, University Department of Home Science, Ranchi University, Ranchi, Jharkhand, India

\section{Glycated hemoglobin (HbA1c) and cardiovascular disease: A review}

\section{Swati Suman and Seema Dey}

DOI: http://dx.doi.org/10.22271/foodsci.2020.v1.i1a.10

\section{Abstract}

Cardiovascular disease (CVD) is a major worldwide health problem and the leading cause of death in industrialized countries. Cardiovascular disease is also the major complication of type 2 diabetes and is responsible for more than $50 \%$ and up to $80 \%$ of deaths in people with diabetes as well as for very substantial morbidity and loss of quality of life. Diabetes can lead to cardiovascular damage in a number of ways. The processes do not develop independently, and each may accelerate or worsen the others. Thus, as diabetes progresses, the heart and blood vessels are exposed to multiple attacks. Cardiovascular disease is spreading fast in the country and is falling in its grip more rapidly due to lack of awareness and facilities. Stating the major reasons for increasing heart problems, there were nine risk factors - heavy doses of alcohol, smoking, excess salt intake in food, fast and fatty food, lack of fruit consumption, no physical activities, tension, blood pressure and diabetes.

The death rates due to cardiovascular disease are either high or appear to be increasing in countries where diabetes is prevalent. The outlook for cardiovascular diseases is alarming when it is considered that the number of people with diabetes worldwide will be more than double by 2025 . Given the global epidemic of diabetes, the double threat of diabetes and CVD is set to explode unless preventative action is taken. It is noteworthy for example that, in some Western populations, CHD rates have declined in the overall population but no consistent decline is seen in people with diabetes. Diabetic patients have a greater likelihood of having dyslipidemia, hypertension, and obesity. Because early detection and prompt treatment may reduce the burden of diabetes and its complications, screening for diabetes may be appropriate under certain circumstances (American Diabetes Association, 2003). Type 2 diabetes mellitus (T2D), together with its common morbidities of hypertension and dyslipidaemia, predisposes to a varied of acute and chronic complications (Gholap et al, 2011). T2D could damage multiorgan system even before symptomatic onset of major complications. It is a dreaded chronic disease, both to an individual and to humanity in the world, especially in Asia (Chan et al, 2009).

Keywords: HbA1c Glycated haemoglobin, CVD Cardio vascular disease, obesity, DM2

\section{Introduction}

Diabetes is a disease which results in high blood sugar level which is also called hyperglycemia, generally results because of lack of insulin secretion from the pancreas of the body. Diabetes is mainly classified in 3 different forms Type I Diabetes Mellitus, Type II Diabetes Mellitus, and Gestational Diabetes. Type $1 \mathrm{DM}$ is insulin dependent and Type 2 is Non-Insulin Dependent Diabetes Mellitus. In patients with DM either due to lack of insulin secretion or decreased sensitivity of tissue to insulin, the metabolism of carbohydrate, fat and protein gets impaired, which leads to an increase in blood glucose level and create a condition called persistent hyperglycemia. Diabetes Mellitus has emerged as a very serious health issue in all over the world and India has distinction of having second largest number of diabetic patients in the country after China, and has become one of the major public health concerns worldwide. (Ramachandran et al, 2010) ${ }^{[26]}$. Now a day's Diabetes Mellitus is considered as a global endemic with a very rapid increase in its prevalence in developed as well as developing countries. (Berry et al, 2007) ${ }^{[2]}$. There are two principle goals in the management of T2DM, namely, to avoid hyperglycemia by maintaining blood glucose levels as close to the normal range as possible $(80-120 \mathrm{mg} / \mathrm{dl})$ and to prevent microvascular and macrovascular complications (Polonsky, 1994; Raskin et al., 1994) ${ }^{[25,27]}$. WHO has declared India as "Diabetic Capital of the world" (Gupta 2002) ${ }^{[8,10]}$. Although the prevalence of both type 1 and type $2 \mathrm{DM}$ is going to increase, type $2 \mathrm{DM}$ is expected to rise more rapidly in future because of increased obesity and reduced activity levels. The chronic complications of DM affect many organ systems and are responsible for the majority of morbidity and mortality associated with the disease. 
The risk of chronic complications increases as a function of the duration of hyperglycemia; they usually become apparent in the second decade of hyperglycemia.

Glycated haemoglobin (HbA1c) is routinely used marker for long term glycemic control. HbA1c predicts the risk for develop diabetic complications in diabetic patients. Now a day's, elevated levels of HbA1c have been regarded as an independent risk factor for cardiovascular disease in subjects with or without diabetes. Each one percent increase in absolute HbA1c shows eighteen percent increase for cardiovascular disease (Selvin et al, 2004) ${ }^{[30,31] .}$.

$\mathrm{HbA1c}$ is a blood sugar test that reflects average plasma glucose over the previous eight to 12 weeks (Nathan et al., 2007) ${ }^{[21]}$. The test can be performed at any time of the day and does not require any special preparation such as fasting. These properties have made it the preferred test for assessing glycaemic control in people with diabetes. More recently, there has been substantial interest in using it as a diagnostic test for diabetes and as a screening test for persons at high risk of diabetes (International Expert Committee Report, 2009) [15]. In 2009, The International Expert Committee recommended the use of HbA1c to diagnose diabetes mellitus with a threshold > 6.5\%.1 The American Diabetes Association (ADA) adopted this recommendation in its position statement entitled, "Standard of medical care in diabetes-2010" (American Diabetes Association, 2010) ${ }^{[1]}$. The estimated prevalence of diabetes in India is 61.3 million people in 2011, which is projected to increase to 101.2 million people in 2030 (David et al., 2011) [5].

The abnormalities like insulin resistance, hyper insulinemia, hyperglycemia, dyslipidemia and hypertension in type 2 diabetes tend to cluster and are often referred to as the "metabolic syndrome (Grundy, 1998) [7]". Elements of metabolic syndrome are strong risk factors for cardio vascular disease (Lamarche et al, 1998) ${ }^{[18]}$. An early intervention to normalize circulating lipids has been shown to reduce cardio vascular complications and mortality (Windler, 2005) ${ }^{[37]}$. Much published data support the conclusion that diabetes puts people at very high risk of coronary heart disease (Folson et al., 2003). Diabetic patients without prior myocardial infarction have high risk of having myocardial infarction at rates comparable to nondiabetic patients with previous myocardial infarction (Haffner et al., 1998) ${ }^{[12]}$. Diabetes patient's face an $11 \%$ increased risk of mortality from ischaemic heart disease (UKPDS 23) (Turner et al., 1998) ${ }^{[35]}$. While those with HbA1c $>8 \%$ face a $150 \%$ increased risk of death from heart disease (Saydah et al., 2009) ${ }^{[29]}$. Deranged lipid levels and insulin levels are the distinctive findings in individuals with insulin resistance, and these have been recognized as increasing risk for cardio metabolic disorders. This dyslipidemia shows distinct pattern of altered lipid metabolism, particularly elevated plasma triglyceride and decreased HDL-cholesterol concentrations and the presence of small and dense LDL particles. The high triglyceride level in blood is associated with insulin resistance and obesity, and that is secondary due to high plasma insulin levels leading to increased esterification of fatty acids in liver resulting in formation of triglycerides. The low HDL level in blood is due to the increased catabolism of HDL molecules augmented by insulin resistance and hypertriglycedemic states (Brunzell and Hokanson 1999) ${ }^{[4]}$. The higher levels of LDL are atherogenic (Mora 2007) ${ }^{[19]}$, however high HDL levels in blood have found to be cardioprotective (Shao and Heinecke 2009) ${ }^{[32]}$. Higher concentration of TGs is also considered a risk factor for cardiovascular diseases. In addition to that, several studies are agreed upon the utility of lipid ratios and found that TC/HDL and LDL/HDL is better predictor of cardiovascular diseases then individual lipid marker (Stampfer, 1991) ${ }^{[33]}$. A study has shown negative correlation between TC/HDL and insulin-mediated glucose disposal highlighting the utility of these ratios in determining the insulin resistance (Ridker et al, 2001) ${ }^{[28]}$. In a study, published in 2013, a strong association has been observed between TG/HDL ratio and insulin resistance in youths, and it was suggested that TG/HDL ratio can be a strong candidate for a surrogate of insulin resistance and atherosclerosis in young population (Oliveira, 2013) ${ }^{[22]}$. This increased risk of developing heart failure could be explained by two mechanisms: the development of endothelial dysfunction, atherosclerosis, and $\mathrm{CHD}$, and direct toxic damage to the myocardium due to chronic hyperglycaemia (Iribarren et al., 2001) ${ }^{[13]}$. Among diabetics, HbA1c is an independent predictor of heart failure even without pre-existing CHD, although the relative incidence rate of heart failure in people with pre-existing CHD is much higher (15.5 for CHD-negative vs. 56.4 for CHD-positive cases per 1000 person) (Pazin-Filho et al., 2008) ${ }^{[23]}$.

Elevated levels of glycated $\mathrm{Hb}$ not only increases incidence of CHD but also extends into subsequent complications of CHD (e.g., congestive heart failure); each 1\% rise in HbA1c results in more coronary events and more hospitalizations due to worsening heart failure (Gerstein et al., 2008) ${ }^{[9]}$. Elevated HbA1c (6.5\% to $7 \%$ ) is associated with poorer prognosis in patients with minimal left ventricular dysfunction (LVEF < 45\%) (Goode et al, 2009) ${ }^{[10]}$.

Type 2 diabetes patients have markedly increased risk of coronary heart disease than similarly dyslipidaemic nondiabetic subjects (Bloomgarden 1998) ${ }^{[3]}$. Most recently, results of the Strong Heart Study indicate that the LDL cholesterol is an independent predictor of cardiovascular disease in patients with diabetes, along with age, albuminuria, fibrinogen, HDL cholesterol (inverse predictor) and percent body fat(inverse predictor) (VanDer et al, 1998). About $20 \%$ of patients with type 2 diabetes have hypertriglyceridaemia or low HDL-C levels (Laakso 1997) ${ }^{[17]}$. These abnormalities, as well as high VLDL-C levels and high total and VLDL-related triglyceride levels, are powerful risk indicators for CAD in patients with type 2 diabetes. In addition, the combination of elevated total cholesterol level, hypertension, and uncontrolled hyperglycemia is implicated in the development of nephropathy. In a study done in Russia, dyslipidaemia was detected in 84\% diabetic patients (Kiriakov et al, 1990) ${ }^{[16]}$. In one European study 40\% were hyperlipidaemic according to the criteria of National cholesterol education programme, (cholesterol and triglyceride greater than $200 \mathrm{mg} / \mathrm{dl}$ ). An additional 23\% showed hypertriglyceridaemia (Stern et al, 1989) ${ }^{[34]}$. In another study hyperlipidaemia was found in $28 \%$ of diabetic patients (Peterson et al, 1991) ${ }^{[24]}$. Diabetes particularly puts younger people $(<55$ years $)$ at risk of stroke and is a cause of poor prognosis post stroke. Raised HbA1c is an independent risk of stroke in people with or without diabetes, unlike CHD this relationship is not linear but it seems more like a threshold dependent association, risk of stroke abruptly increases at HbA1c $>7 \%$ regardless 
of diagnosis of diabetes. Although the people with HbA1c $6.5 \%-7 \%$ are more likely to be undiagnosed diabetics, show significantly increased risk of stroke after adjustment for classic risk factors like hypertension and smoking (Myint et al., 2007) ${ }^{[20]}$.

Diabetes increases risk of PVD by more than two folds, and is cause of $70 \%$ non-traumatic amputations. It is a manifestation of CVD which is not acutely fatal but is associated with serious risk of CHD and stroke. HbA1c is positively associated with low ankle-brachial index and symptomatic PVD (Selvin et al, 2006) ${ }^{[30,31]}$, although it is not only due to macrovascular disease but a great element of microvascular disease is also present in processes like neuropathy, foot ulceration, and amputations. The impact of cardiovascular disease in diabetes is exacerbated even further by the earlier age of onset of type II diabetes which is now reaching down even to children and adolescents, and carries the threat of early onset of CVD. In addition advances in insulin therapy have improved the life expectancy of people with type I diabetes and each year of prolonged life increases the likelihood of cardiovascular complications. Diabetes leads to cardiovascular damage by a number of mechanisms, each of which in turn may accelerate or worsen the others. It belongs to a special risk category as it has so marked an effect on cardiovascular risk. As well as being a risk factor in its own right, diabetes is associated with a higher prevalence of other common risk factors such as hypertension and dyslipidemia, and, these risk factors, in turn, have a more harmful effect in the presence of diabetes. For each risk factor present, the risk of cardiovascular death is about three times greater in people with diabetes compared to those without diabetes.

Regular blood sugar checkups and control of HbA1c level to normal range decreases the risk of heart health problems such as heart failures and coronary heart disease. Prolong high level of HbA1c level can show adverse effects on heart and can lead to other complications.

\section{Reference}

1. American Diabetes Association. Position Statement; Standards of Medical Care in Diabetes - 2010. Diabetes Care 2010;33(Suppl-1):S11-61.

2. Berry C, Tardif JC, Bourassa MG. Coronary heart disease in patients with diabetes: part I: recent advances in prevention and noninvasive management. J Am Coll Cardiol 2007;49:631-642.

3. Bloomgarden ZT. International Diabetes Federation meeting (type 2 diabetes: its prevalence, causes, and treatment). Diabetes Care 1998;21(5):860-865.

4. Brunzell JD, Hokanson JE. Dyslipidemia of central obesity and insulin resistance. Diabetes Care 22 Suppl 1999;3:C10-13.

5. David RW, Leonor G, Clara W, Jonathan S. IDF Diabetes Atlas: Global estimates of the prevalence of diabetes for 2011 and 2030. Diabetes research and clinical practice 2011;94(3):311-321.

6. Folsom AR, Chambless LE, Duncan BB, Gilbert AC. Pankow JS. Prediction of coronary heart disease in middle-aged adults with diabetes. Diabetes Care 2003;26:2777-84.

7. Grundy SM. Multifactorial causation of obesity: implication for prevention. Am J Clin. Nutr 1998;67:5635-5725.

8. Gupta V. Diabetes in Elderly patients. JK practitioner
2002;91(4):258-259.

9. Gerstein HC, Swedberg K, Carlsson J, Mcmurray JJV, Michelson EL, Olofsson B. The haemoglobin A1c level as a progressive risk factor for cardiovascular death, hospitalization for heart failure, or death in patients with chronic heart failure. Arch Intern Med 2008;168:1699-704.

10. Goode KM, John J, Rigby AS, Kilpatrick Gupta V. Diabetes in Elderly patients. JK practitioner 2002;91(4):258-259.

11. Gu K, Cowie CC, Harris MI. Diabetes and decline in heart disease mortality in US adults. J Am Med Assoc 1999;281:1291-1297.

12. Haffner SM, Lehto S, Rönnemaa T, Pyörälä K, Laakso M. Mortality from coronary heart disease in subjects with type 2 diabetes and in non-diabetic subjects with and without previous myocardial infarction. N Engl J Med 1998;339:229-234.

13. Iribarren C, Karter AJ, Go AS, Ferrara A, Jennifer Y, Sidney S. Glycemic Control and Heart Failure among adult patients with diabetes. Circulation 2001;103:2668-73.

14. International Diabetes Federation. Diabetes and Cardiovascular Disease: Time to Act. International Diabetes Federation, Brussels 2001.

15. International Expert Committee report on the role of the A1C assay in the diagnosis of diabetes. Diabetes Care 2009;32:1327-1334.

16. Kiriakov A, Koev D, Kavrykova IU. The phenotypic characteristics and incidence of dyslipidaemias in diabetic patients. Probl Endokrinol, Mosk 1990;36(6).

17. Laakso M, Pyorala K. Age of onset and type of diabetes. Diabetes Care 1985;8:114-117.

18. Lamarche B, Tchernf A, Mauriege P, Canlin B, Dagenius GR, Despress JP. Fasting insulin and apolipoproteinB levels and low density lipoprotein size as risk factors for ischemic heart disease. JAMA 1998;279:1955-1961.

19. Mora S. LDL particle subclasses, LDL particle size, and carotid atherosclerosis in the Multi-Ethnic Study of Atherosclerosis (MESA). Atherosclerosis 2007;192(1):211-217.

20. Myint PK, Sinha S, Wareham NJ, Bingham S, Luben RN, Welch AA. Glycated haemoglobin and risk of stroke in people without known diabetes in European prospective investigation into cancer (EPIC) - Norfolk prospective population study, A threshold relationship? Stroke 2007;38:271-5.

21. Nathan DM, Turgeon H, Regan S. Relationship between glycated haemoglobin levels and mean glucose levels over time. Diabetologia 2007;50:2239-2244.

22. Oliveira AC. Is triglyceride to high-density lipoprotein cholesterol ratio a surrogates for insulin resistance in youth? Health 2013;5(3):481-485.

23. Pazin-Filho A, Kottgen A, Russell SD, Bertoni AG, Slevin E, Rosamond WD. HbA1c as a risk factor for heart failure in persons with diabetes: the atherosclerosis risk in communities (ARIC) study. Diabetologia 2008;51:2197-204.

24. Peterson JR, Pettigrew AR, Dominiczak MH, Small M. Screening for hyperlipidaemia in diabetes mellitus. Relationship to glycaemic control. Ann Clin Biochem 1991;28:354.

25. Polonsky WH. Psychosocial issues in diabetes mellitus. 
In $\mathrm{R}$ Gatchel and E Blanchard (Eds.) Psychophysiological disorders: Research and clinical applications. Washington DC: American Psycholoical Association 1994.

26. Ramachandran A, Ma RCW, Snehlatha C. Diabetes in Asia Lancet 2010;375:408-18.

27. Raskin P. Medical Management of non-insulin dependent (Type II) diabetes. nAlexandria VA: American Diabetes Association. Folsom A.R, Chambless L.E., Duncan B.B. and Gilbert A.C. (2003) Pankow JS. Prediction of coronary heart disease in middle-aged adults with diabetes. Diabetes Care 1994;26:2777-84.

28. Ridker PM, SMJRN. Novel risk factors for systemic atherosclerosis: A comparison of $\mathrm{C}$ - reactive protein, fibrinogen, homocysteine, lipoprotein (a), and standard cholesterol screening as predictors of peripheral arterial disease. JAMA 2001;285(19):2481-2485.

29. Saydah S, Tao M, Imperatore G, Gregg E. GHb Level and subsequent mortality among adults in the U.S. Diabetes Care 2009;32:1440-6.

30. Selvin E, Marinopoulos S, Power NR. Meta-analysis: glycosylated haemoglobin and cardiovascular disease in diabetes mellitus. Ann Intern 2004;14:421-31.

31. Selvin E, Coresh J, Wattanakit K, Steffes MW, Sharrett R. HbA1c and Peripheral arterial disease in diabetes, The ARIC Study. Diabetes Care 2006;29:877-82.

32. Shao B, Heinecke JW. HDL, lipid peroxidation, and atherosclerosis. Journal of Lipid Research 2009;50(4):599-601.

33. Stampfer MJ, Sacks FM, Salvini S, Willett WC, Hennekens $\mathrm{CH}$. A prospective study of cholesterol, apolipoproteins, and the risk of myocardial infarction. N Engl J Med 1991;325:373-381.

34. Stern MP, Patterson JK, Haffner SM, Hazuda HP, Mitchell BD. Lack of awareness and treatment of hyperlipidaemia in type 2 diabetes in a community survey. JAMA 1989;262(3):360.

35. Turner RC, Millns H, Neil HAW, Straton IM, Mathews DR, Holman RR. Risk factors for coronary artery disease in non-insulin dependent diabetes mellitus: United Kingdom prospective diabetes study (UKPDS:23). BMJ 1998;316:823-8.

36. Van der does FEE, Neeling JND, Snoek FJ. Randomized study of two different target levels of glycemic control within the acceptable range in type 2 diabetes (Effects on well-being at 1 year). Diabetes Care 1998;21(12):2085-2093.

37. Windler E. What is the consequence of an abnormal lipid profile in patients with type 2 diabetes or the metabolic syndrome? Atheroscler. Suppl 2005;6:11-14. 